\title{
A NEW GENERALIZED REFINEMENTS OF
} YOUNG'S INEQUALITY AND APPLICATIONS

\section{MOHAMED Amine IGHACHANE AND MOHAMED AKKOUCHI}

Abstract. In this work, by the weighted arithmetic-geometric mean inequality, we show if $a, b>$ 0 and $0 \leqslant v \leqslant 1$. Then for all positive integer $m$, we have

$$
\begin{aligned}
\left(a^{v} b^{1-v}\right)^{m}+ & r_{0}^{m}\left((a+b)^{m}-2^{m}(a b)^{\frac{m}{2}}\right) \\
& +r_{m}\left[\left((a b)^{\frac{m}{4}}-b^{\frac{m}{2}}\right)^{2} \chi_{\left(0, \frac{1}{2}\right]}(v)+\left((a b)^{\frac{m}{4}}-a^{\frac{m}{2}}\right)^{2} \chi_{\left(\frac{1}{2}, 1\right]}(v)\right] \\
\leqslant & (v a+(1-v) b)^{m},
\end{aligned}
$$

where $r_{0}=\min \{v, 1-v\}, r_{m}=\min \left\{2^{m} r_{0}^{m},\left(1-r_{0}\right)^{m}-r_{0}^{m}\right\}$ and $\chi_{I}(v)$ the characteristic function. This inequality provides a generalization of an important refinement of the Young inequality obtained by J. Zhao and J. Wu. As applications we give some new generalized refinements of Young type inequalities for the determinants, $p$-norms and traces, of positive $\tau$-measurable operators.

Mathematics subject classification (2020): 26D07, 26D15, 46L52, 47A63.

Keywords and phrases: AM-GM inequality, Young's inequality, von Neumann algebras, determinants, $p$-norms, trace.

\section{REFERENCES}

[1] Y. Al-Manasrah and F. Kittaneh, A generalization of two refined Young inequalities, Positivity 19 (2015), 757-768.

[2] Y. Al-ManasRah And F. KitTAneh, Further generalization refinements and reverses of the Young and Heinz inequalities, Results, Math. 19 (2016), 757-768.

[3] D. CHOI, A generalization of Young-type inequalities, Math. Inequalities Appl. 21 (2018), 99-106.

[4] B. Fuglede, Rv. Kadison, On determinants and a property of the trace in finite factors, Proc. Nat. Acad. Sci. 37 (1951), 425-431.

[5] B. Fuglede, Rv. Kadison, Determinants theory in finite factors, Ann. Math. 55 (1952), 520-530.

[6] M. Hajmohamadi, R. Lashkaripour, M. Bakherad, Some extensions of the Young and Heinz. inequalities for matrices, Bulletin of the Iranian Mathematical Society, 44 (4), 2018, 977-990.

[7] Y. HAN, Some determinant inequalities for operators, Linear and Multilinar Algebra 67 (2017), 1-12.

[8] O. HirZallah, AND F. KitTANeH, Matrix Young inequalities for the Hilbert-Schmidt norm, Linear Algebra Appl. 308 (2000), 77-84.

[9] G. LAROTONDA, The case of equality in Young's inequality for the s-numbers in semi-finite von Neumann algebras, J. Operator Theory 81 (1), (2019), 157-173.

[10] F. Kittaneh, And Y. Al- Manasrah, Improved Young and Heinz inequalities for matrices, J. Math. Anal. Appl. 36 (2010), 292-269.

[11] M. SabABheh And D. Choi, A complete refinement of Young's inequality, J. Math. Anal. Appl. 440, 1, (2016), 379-393.

[12] J. SHAO, Generalization of refined Young inequalities and reverse inequalities for $\tau$-measurable operators, Linear and Multilinar Algebra (2019), 1563-5139. 
[13] G. Pisier, Q. XU, Noncommutative $L_{p}$ spaces, Handbook of the geometry of Banach spaces 2 (2003), 1459-1517.

[14] J. Zhou Y. WAng, T. WU, A Schwarz inequality for $\tau$-measurable operators $A^{*} X B^{*}$, J. Xinjiang Univ. Naatur. Sci. 1(2009), 69-73.

[15] J. ZhaO AND J. WU, Operator inequalities involving improved Young and its reverse inequalities, J. Math. Anal. Appl. 421 (2015), 1779-1789. 